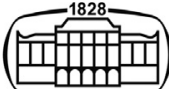

AKADÉMIAI KIADÓ

\title{
Chromatographic fingerprinting and quantitative analysis for the quality evaluation of Xinfeng capsule
}

\section{Acta Chromatographica}

33 (2021) 1, 37-43

DOI:

10.1556/1326.2020.00743 The Authors

\section{ORIGINAL RESEARCH} PAPER

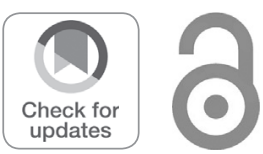

\author{
LEILEI GAO ${ }^{4}$, FANG WANG $^{1,2,3 *}$ (1) and MEI MENG ${ }^{3 * *}$ (])
}

${ }^{1}$ College of Biotechnology and Pharmaceutical Engineering, West Anhui University, Lu'an, 237012, China

2 The Provincial 2011 Collaborative Innovation Center of Anhui-Dendrobium Huoshanense Industrialization Exploitation Collaborative Innovation Center, Lu'an, 237012, China

${ }^{3}$ Key Laboratory of Xinan Medicine, Ministry of Education, State Administration of Traditional Chinese Medicine TCM Preparation Level 3 Laboratory, The First Affiliated Hospital of Anhui University of Chinese Medicine, Hefei, Anhui, 230031, China

${ }^{4}$ School of Pharmacy, Anhui University of Chinese Medicine, Hefei, 230012, China

Received: November 6, 2019 - Accepted: February 3, 2020

Published online: April 16, 2020

\footnotetext{
*Corresponding author. College of Biotechnology and Pharmaceutical Engineering, West Anhui University, Lu'an, 237012, China.

E-mail: wang1990f@sina.cn

${ }^{* *}$ Corresponding author. E-mail: great1005@126.com
}

\section{KEYWORDS}

HPLC, Xinfeng capsule, fingerprint, identification, quality control

\begin{abstract}
A simple, accurate and sensitive method of high performance liquid chromatography (HPLC ) with diode array detector was established to identify Xinfeng capsules and systematically evaluated its quality, based on chromatographic fingerprint integrated with the similarity analysis, hierarchical cluster analysis and the quantitative analysis of multi-components by single marker (QAMS). In this study, 18 peaks were selected as the common peaks to evaluate the similarities among different batches (S1-S10) of Xinfeng capsules samples, which were manufactured in the First Affiliated Hospital of Anhui University of Chinese Medicine with a three-year span. Compared to control fingerprint, the similarities values for 10 batches of samples were more than 0.90 . Moreover, by analyzing the reference of astragalus, the chromatogram of astragalus was developed, and 10 common peaks of astragalus were identified. More importantly, simultaneous quantification of three markers in Xinfeng capsule, including Calycosin-7-glucoside, calycosin and Formononetinaldehyde was performed, the three constituents showed good regression $(R>0.999)$ within linear ranges, and their recoveries were within the range of $97.6-101.5 \%$. The validation results showed that the developed method was specific, accurate, precise and robust. This study demonstrated that the developed method offers an efficient, reliable and practical approach for systematic quality evaluation of Xinfeng capsule.
\end{abstract}

\section{INTRODUCTION}

Rheumatoid arthritis (RA) is a chronic inflammatory disease characterized by hyperplasia of synovial lining cells, infiltration of mononuclear cells, and abundant angiogenesis in affected joints, all of which ultimately lead to the destruction of cartilage and bone $[1,2]$. Arthritis mainly leads to joint damage, deformities, loss of function, systemic complications including lung disease and coronary artery disease, and permanent disability often within the first 2 years of disease onset $[3,4]$. Xinfeng capsule, a hospital preparation of the First Affiliated Hospital of Anhui University of Chinese Medicine (Anhui medicine system Z20050062) consists of four herbs, which are Astragalus membranaceus (Fisch) Bge. (referred as astragalus), Coix lacryma-jobi L.var.ma-yuen (Roman.) Stapf (referred as myotonin), Tripterygium wilfordii Hook. $f$. (referred as thunder god vinen), ScolopendrasubspinipesmutilansL.Koch 
(referred as centipede) $[5,6]$, and has been utilized in the treatment of RA for about 20 years. Clinical studies have identified that Xinfeng capsule has unique advantages and less side effects as one of the ideal Traditional Chinese Medicine (TCM) on rheumatoid arthritis therapy [7].

TCM plays a significant role in the health care of Chinese people in ancient time and has become increasingly popular all over the world. Contrary to modern pharmacology that often focuses on the single chemical entity aimed at a specific single target, TCM always refers to complex mixtures and one herb usually contains hundreds of chemically different components. Their curative effects are principally based on the synergic effect of their multi-targeting and multi-ingredient preparations [8]. However, questions of quality control are a problem which has restricted the development of TCM and their worldwide commercial acceptance. Therefore, it is of great importance to establish a suitable scientific quality control system for TCM. The technique of TCM chromatographic fingerprinting is playing an increasingly important role in the quality control of TCM, and has proven to be practical and scientific [9].

Recently, the chromatographic fingerprint technique has been introduced as a tool to evaluate the quality of herbal samples or their derived products [10-13]. This technique has been accepted by the WHO and European Medicines Agency (EMEA) as a strategy for the quality assessment of herbal medicines [14] and gained more and more attention, because it emphases on the characterization of the complete sample composition. Several methods, such as high-performance thin layer chromatography (HPTLC) [15-18], gas chromatography (GC) [19-21], capillary electrophoresis (CE) [22-24], infrared spectroscopy (IR) [25], nuclear magnetic resonance (NMR) [26-29], mass spectrometry (MS) $[30,31]$ and high-performance liquid chromatography (HPLC) [32-34] are applied for fingerprinting. Among these methods mentioned above, HPLC is the most popular method and widely used for the fingerprint analysis [35-38].

Although there have been some reports concerning the quality control of Xinfeng Capsule, most of the reports have been limited to the qualitative and quantitative analyses of several components rather than using chemical fingerprinting [5]. The study of relationship between each chromatographic peak from the herbs and preparation is of great significance. In research into compound preparations, it is important to note the origin of each chromatographic peak of the preparation fingerprint and study the change in the chromatographic peaks of each herb when the medicinal herbs are decocted both separately and together. The investigation of the correlation is fundamental and a basis of preparation quality control. There was, unfortunately, no information in the literature about the use of HPLC fingerprint for analysis of this preparation. Therefore, it is significant to establish a HPLC-DAD method for fingerprint analysis of Xinfeng capsule.

Based on the principle rule of "sovereign-minister-assistant-courier," astragalus act as sovereign herb in Xinfeng capsule. Importantly, astragalus is the main therapeutic drug and plays a leading role in the preparation. Thus, astragalus was mainly researched and selected as the control raw medicinal material. At equal pace, three bioactive constituents of the sovereign herb astragalus (alycosin-7-glucoside, calycosin and Formononetin) were simultaneously determined for the first time. All in all, in the present study, a simple, accurate and sensitive method was developed to control the quality of Xinfeng capsule in different batches.

\section{EXPERIMENTAL}

\section{Materials and reagents}

Calycosin-7-glucoside, calycosin and Formononetin were purchased from National Institutes for Food and Drug Control. The purity of each standard was over $98 \%$ and suitable for HPLC determination. The herbs (astragalus, myotonin, thunder god vine, and centipede) for the proposed study were purchased from Lejialaopu (Hefei, Anhui), and authenticated by Lihua Li Professor (Department of the First Affiliated Hospital of Anhui University of Chinese Medicine). Xinfeng capsules of 10 different batches (batch No. 20120625, 20120825, 20120925, 20121025, 20121124, 20130112, 20130530, 20130902, 20140122, 20140220) were collected from the First Affiliated Hospital of Anhui University of Chinese Medicine. HPLC-grade acetonitrile and HPLC-grade methanol were obtained from Sinopharm Chemical Reagent (Sinopharm Chemical Reagent Co., Ltd, China). Ultrapure water was prepared by a Milli-Q50 SP Reagent Water System (Millipore Corporation, MA, USA). Other reagents were all of analytical grade were purchased from Shanghai Zhenqi chemical reagent (Shanghai, China).

\section{Apparatus and chromatographic conditions}

Analyses were performed on an Agilent 1260 series HPLC system consisting of a quatpump, an online degasser, an auto plate-sampler, a column oven, and a diode array detector (DAD). All separation steps were carried out on an Agilent Eclipse plus $\mathrm{C}_{18}$ column $(4.6 \mathrm{~mm} \times 250 \mathrm{~mm}, 5 \mu \mathrm{m})$. The mobile phase was composed of $1 \%$ phosphoric acid aqueous solution (A) and acetonitrile (B) in gradient elution mode. The flow rate of the mobile phase was kept at $1 \mathrm{~mL} /$ min and the gradient of acetonitrile (B) was changed as follows: $0-10 \mathrm{~min}, 5 \%$; $10-30 \mathrm{~min}, 5-15 \%$; 30-55 $\mathrm{min}, 15-$ 25\%; 55-75 min, 25-35\%; 75-95 min, 35-45\%; 95-110 min, 245-65\%; 110-130 min, 65-90\%; 130-140 min, 90-95\%; 140-145 min, 95-100\%; and 145-155 min, 100\%. During fingerprint analysis, the wavelength was set at $210 \mathrm{~nm}$, which exhibited the vast majority of chromatographic peaks. The column temperature was kept at $35{ }^{\circ} \mathrm{C}$ and the sample injection volume was $10 \mu \mathrm{L}$.

\section{Sample preparation}

Standard solutions. The three reference compounds were accurately weighed and dissolved in methanol at concentrations Calycosin-7-glucoside $0.21 \mathrm{mg} / \mathrm{mL}$, calycosin 0.1896 
$\mathrm{mg} / \mathrm{mL}$ and formononetin $0.08 \mathrm{mg} / \mathrm{mL}$ to obtain stock standard solutions. The solutions were stored at $4{ }^{\circ} \mathrm{C}$ and kept in a dark place.

Sample solutions. The content powder of Xinfeng capsules ( $4.0 \mathrm{~g})$ in different batches samples were actually weighted, it was then transferred into a conical flask with cover $(100 \mathrm{~mL})$ containing $40 \mathrm{~mL}$ of methanol, extracted in an ultrasonic water bath for $40 \mathrm{~min}$ at room temperature and filtered through a filter paper. The filtrate was concentrated to dry and further diluted with methanol. It was then transferred to a $2 \mathrm{~mL}$ volumetric flask and diluted to volume with methanol; they were for the analysis (Nos. 1, 2, 3, 4, 5, 6, 7, 8, 9, and 10). All the solutions were stored at $4{ }^{\circ} \mathrm{C}$ and kept in a dark place. The sample solutions were filtered through a 0.22 $\mu \mathrm{m}$ nylon filter membrane before HPLC analysis.

Astragalus and blank herb solutions. According to the proportion of Xinfeng Capsule prescription each single herb and blank were accurately weighed. Based on the Xinfeng Capsule technology system preparation steps and methods, dry extracts of astragalus and blank were obtained. According to extract yield and each group of prescription medicines in the amount ratio, respectively, amount of each single herbs and blank herb extract were weighed, and then referring to the sample solution processing method astragalus and blank herb solutions were obtained.

\section{Data analysis}

Chromatographic fingerprint data were analyzed by the professional software Similarity Evaluation System for Chromatographic Fingerprint of TCM (Version 2004A), which was recommended by China Food and Drug Administration (CFDA). The correlation coefficient of the samples was calculated and similarity comparison was performed between individual chromatogram and the common chromatogram from the samples tested.

\section{RESULTS AND DISCUSSION}

\section{Optimization of chromatographic conditions}

Initially, different compositions of mobile phase (methanolphosphoric acid, acetonitrile-phosphoric acid, acetonitrile$0.1 \%$ phosphoric acid, acetonitrile- $0.2 \%$ phosphoric acid, acetonitrile- $0.3 \%$ phosphoric acid,) were tested. As a result, acetonitrile- $0.1 \%$ phosphoric acid in the gradient mode was adopted because of the more stable baseline, the better separation and the more characteristic peaks in the chromatograms.

In addition, three column temperatures, 25, 30, and $35{ }^{\circ} \mathrm{C}$, were also tested. Eventually, the optimal condition was determined as $35{ }^{\circ} \mathrm{C}$. In a full-scan chromatogram depicted in Fig. 1 not only lots of peaks could be detected at $210 \mathrm{~nm}$, but also 3 compounds shown good absorptivity than at other wavelengths by comparing with 234, 254, 280, 300 , and $320 \mathrm{~nm}$. However, more interferences were also

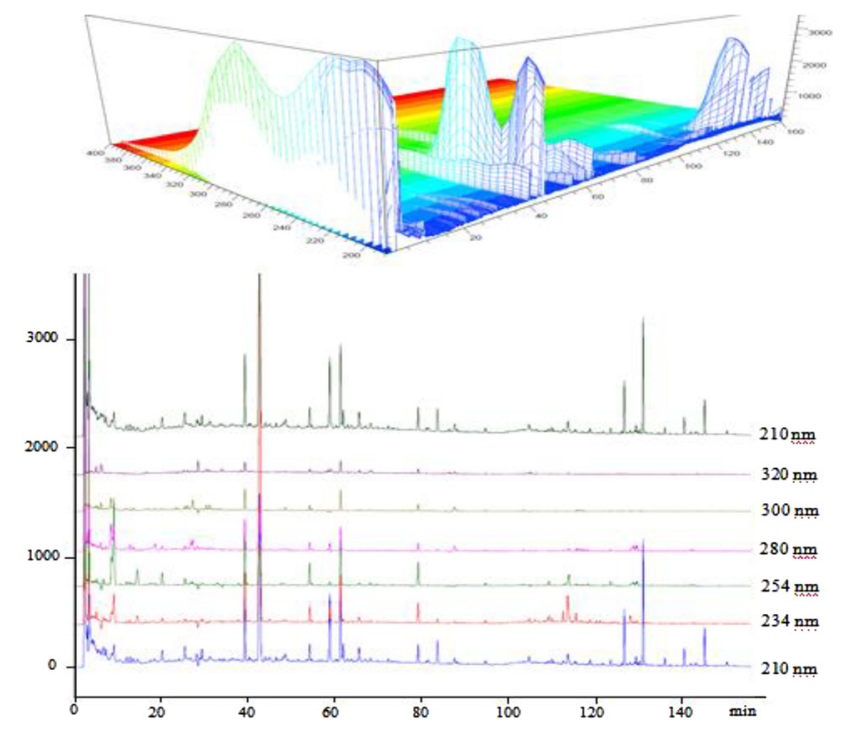

Figure 1. HPLC chromatogram of XFC extract visualized by 3D, $210,234,254,280,300$ and $320 \mathrm{~nm}$, respectively

observed in those wavelengths, which was smaller than 210 $\mathrm{nm}$. In order to reflect as much as informative chemical characteristics of Xinfeng capsule, the most appropriate wavelength was set as $210 \mathrm{~nm}$.

\section{Optimization of sample extraction}

In terms of the extraction solvent, extraction method and extraction time were all investigated. The solvent of methanol was found to have much more peaks with higher response and better peak shape than ethanol. Then, various solvent ratios of methanol $(60,80$, and $100 \%)$ were evaluated, and $100 \%$ methanol was chosen for its highest extraction yields. Finally, ultrasonication (30, 40, and 60 $\mathrm{min})$ and heat reflux (30, 40, and $60 \mathrm{~min})$ extraction methods were examined. The results showed that ultrasonication $40 \mathrm{~min}$ was the most effective by comparing to the peak areas of analytes in HPLC chromatograms. Thus, $100 \%$ methanol ultrasonic extracting for $40 \mathrm{~min}$ was confirmed to be the optimal extraction methods.

\section{Method validation of quantitative analysis}

Methanol stock solutions containing the three analytes were prepared, and the chemical structures of three active components were showed in Fig. 2. Different concentrations of the three analytes solution were analyzed, and then the calibration curves were generated by peak area $(y)$ versus the concentrations of each analyte $(x)$. The calibration curves and ranges of the three components were presented in Table 1 and Fig. 3. And, all of the analytes achieved good linearity $\left(r^{2}>0.999\right)$ within test ranges.

The results of apparatus precision of the three analytes and their relative standard deviation (RSD) values were less than $2.0 \%(n=6)$. Besides, repeatability studies of this method proved that this assay has good reproducibility with RSD less than $2.6 \%(n=6)$ for the three analytes. The 
<smiles>COc1ccc(-c2coc3cc(O)ccc3c2=O)cc1O</smiles>

calycosin (1)

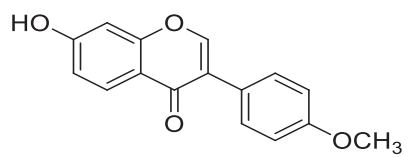

Formononetin (3)<smiles>COc1ccc(-c2coc3cc(OC4OC(OC)[C@@H](O)C(O)C4O)ccc3c2=O)cc1O</smiles>

Calycosin-7-glucoside (2)

Figure 2. The chemical structures of the investigated compounds

Table 1. Linear regression data $(n=6)$

\begin{tabular}{cccc}
\hline Components & Regression equation & $\begin{array}{c}\text { Correlation } \\
\text { coefficient }\left(r^{2}\right)\end{array}$ & $\begin{array}{c}\text { Linear } \\
\text { range } \\
(\mu \mathrm{g})\end{array}$ \\
\hline $\begin{array}{c}\text { Calycosin-7- } \\
\text { glucoside }\end{array}$ & $y=4472.1 x-1.2128$ & 1 & $0.42-$ \\
Calycosin & $y=5838.2 x-41.918$ & 0.9998 & 2.52 \\
& & & $0.38-$ \\
Formononetin & $y=5326.5 x-23.241$ & 0.9999 & 3.03 \\
& & & $0.16-$ \\
\end{tabular}

stabilities of the sample solutions were analyzed at $0,2,4,8$, 12,24 , and $48 \mathrm{~h}$ after storage for two days at room temperature. It was found that the sample solutions were stable within $48 \mathrm{~h}(\mathrm{RSD}<3.21 \%)$.

Recovery test was used to evaluate the accuracy of this method. It was determined by adding the standards with three different levels (high, middle and low) to $2.0 \mathrm{~g}$ of Xinfeng capsule (batch no. 20120925). Then the samples were extracted and analyzed with the proposed method, and triplicate experiments were performed at each level. The average recoveries were estimated by the formula: recovery $(\%)=[$ (amount found-original amount $) /$ amount added] $\times 100 \%$. The average recoveries of the three compounds were in the range of 94.4-107.2\%, with RSD < $3.91 \%$. Therefore, the above parameters demonstrated that the method was considered to be accurate for simultaneously quantitation of the three compounds.

\section{Quantitation of compounds in Xinfeng samples}

For the simultaneous determination of three compounds in Xinfeng capsule from different batches, the developed HPLC method was used to compare to the retention times and online UV spectra with those of standards (Fig. 4). The contents of the 3 analytes in the samples were then calculated. As shown in Table 2, the results indicated that the content variability was quite significant. The content of calycosin-7-glucoside ranged from 74.47 to $97.08 \mu \mathrm{g} / \mathrm{g}$, calycosin ranged from 105.01 to $110.48 \mu \mathrm{g} / \mathrm{g}$, while the content of formononetin ranged from 36.39 to $37.70 \mu \mathrm{g} / \mathrm{g}$.

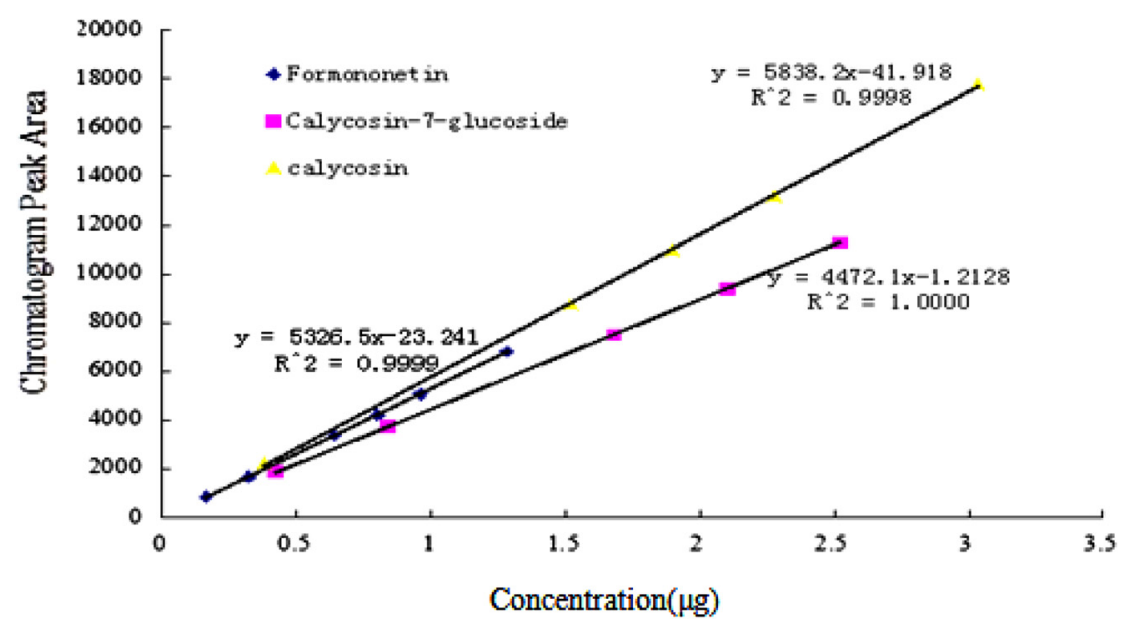

Figure 3. Calibration curves of standards of calycosin-7-glucoside, calycosin and formononetin 


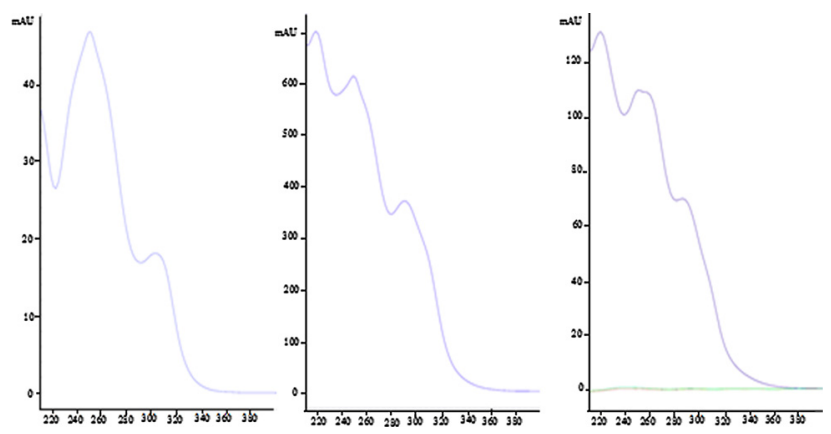

Figure 4. The UV spectrum of three components in Xinfeng capsule

Table 2. Content $(\mu \mathrm{g} / \mathrm{g})$ of the 3 compounds in Xinfeng capsule (10 batches)

\begin{tabular}{ccccc}
\hline & \multicolumn{3}{c}{ Content $(\mu \mathrm{g} / \mathrm{g})$} & $\begin{array}{c}\text { Total } \\
\text { content } \\
(\mu \mathrm{g} / \mathrm{g})\end{array}$ \\
\cline { 2 - 4 } No & $\begin{array}{c}\text { Calycosin-7- } \\
\text { glucoside }\end{array}$ & Calycosin & Formononetin & \\
\hline 20140220 & 96.34 & 110.48 & 37.37 & 244.19 \\
20120625 & 95.42 & 109.85 & 37.60 & 242.87 \\
20130112 & 97.08 & 108.61 & 37.70 & 243.39 \\
20140122 & 94.47 & 105.01 & 36.62 & 236.10 \\
20121025 & 96.70 & 105.26 & 36.66 & 238.61 \\
20130530 & 95.12 & 106.34 & 36.67 & 238.13 \\
20120925 & 95.16 & 106.41 & 37.03 & 238.59 \\
20130902 & 95.88 & 107.36 & 36.63 & 239.88 \\
20121124 & 95.25 & 106.53 & 37.33 & 239.11 \\
20120825 & 96.56 & 109.07 & 36.39 & 242.02 \\
\hline
\end{tabular}

\section{Method validation of fingerprint}

Apparatus precision was assessed by analyzing the same solution of three standards six consecutive times within one day. The results were expressed as relative standard deviations, and appeared that the RSD values of relative retention time (RRT) and relative peak area (RPA) did not exceed 0.26 and $5.10 \%$, respectively.

To confirm the repeatability of the method, six independently prepared solutions from the same sample (batch No: 20120925) were analyzed. The RSD values of relative retention time and relative peak area did not exceed 0.33 and $6.33 \%$, respectively.

The stabilities of the sample solutions were analyzed at 0 , $2,4,8,12$, and $24 \mathrm{~h}$ after storage for one day at room temperature. The RSD values of relative retention time and relative peak area did not exceed 0.59 and $7.64 \%$, respectively. It was found that the sample solution was stable within the tested time period.

\section{HPLC-DAD fingerprint analysis}

To perform fingerprinting analysis, the chromatograms of different batch samples have to be standardized. The process of standardization included the selection of "common peaks" in chromatograms and the normalization of retention times of all the common peaks. To standardize the fingerprint, 10 samples from different batches were analyzed with the developed procedure. Peaks existing in all batches of samples were assigned as "common peaks" for Xinfeng capsule. Chromatograms of these samples were shown in Fig. 5A, and the reference fingerprint of Xinfeng Capsule was shown in Fig. 5B. More than 30 peaks were observed in the chromatogram of Xinfeng capsule extracts, among which 18 peaks were defined as common peaks because they occurred in all samples. Peaks 3, 7, 10 in the chromatogram were identified to be compounds 1,2 , and 3 , respectively, by comparing to the chromatogram of standard solution.

Correlation coefficient was used as a measure for similarity analysis. The chromatogram (batch No: 20120925) of a standardized extract was recommended to serve as the reference profile. In this work, similarity analysis was performed by comparing between chromatogram of each sample and common chromatogram via Chromatographic Fingerprint Similarity Evaluation software. As listed in Table 3, the similarity index was in a range of 0.911-0.990, which suggested that the capsules from different bathes shared the similar chromatographic patterns as shown in Fig. 5.

\section{Identification fingerprint "common peaks" by the reference of Astragalus}

Astragalus and its negative solution of $10 \mu \mathrm{L}$ were for injection, three peaks were identified by comparing their retention times and UV spectra with those of the corresponding standards. Moreover, we also obtained the relationship between common peaks and Astragalus (Fig. 6). Astragalus had most contribution to HPLC fingerprint of Xinfeng Capsule, and

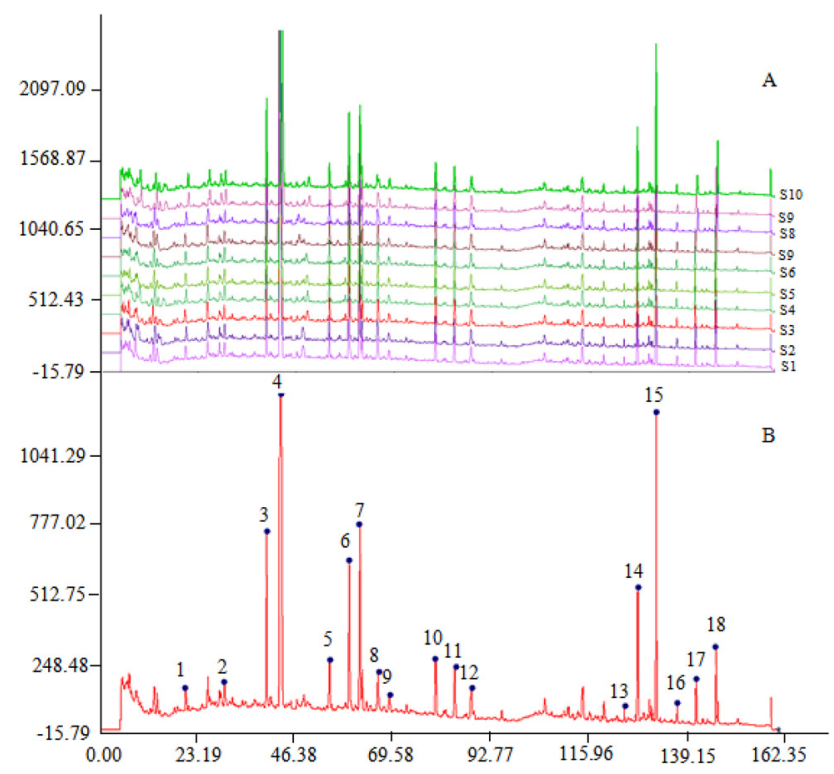

Figure 5. HPLC fingerprint of 10 batches of samples A), and the reference chromatogram B) that was obtained using the similarity evaluation system for chromatographic fingerprint of traditional Chinese medicine software. All chromatograms were detected at the wavelength of $220 \mathrm{~nm}$. The peaks marked with 1-18 in the chromatogram represent 18 common peaks. The peaks marked with 3, 7, 10 are Calycosin-7-glucoside, calycosin and formononetin, respectively 
Table 3. The similarities of Xinfeng capsule from different batches

\begin{tabular}{|c|c|c|c|c|c|c|c|c|c|c|c|}
\hline & 1 & 2 & 3 & 4 & 5 & 6 & 7 & 8 & 9 & 10 & Reference \\
\hline 1 & 1 & 0.935 & 0.978 & 0.913 & 0.996 & 0.995 & 0.938 & 0.990 & 0.911 & 0.935 & 0.950 \\
\hline 2 & 0.935 & 1 & 0.947 & 0.972 & 0.944 & 0.942 & 0.994 & 0.951 & 0.969 & 0.928 & 0.987 \\
\hline 4 & 0.913 & 0.972 & 0.960 & 1 & 0.927 & 0.926 & 0.971 & 0.928 & 0.991 & 0.95 & 0.985 \\
\hline 5 & 0.996 & 0.944 & 0.984 & 0.927 & 1 & 0.999 & 0.946 & 0.992 & 0.925 & 0.941 & 0.960 \\
\hline 6 & 0.995 & 0.942 & 0.984 & 0.926 & 0.999 & 1 & 0.943 & 0.991 & 0.923 & 0.938 & 0.957 \\
\hline 8 & 0.990 & 0.951 & 0.976 & 0.928 & 0.992 & 0.991 & 0.954 & 1 & 0.925 & 0.944 & 0.962 \\
\hline 9 & 0.911 & 0.969 & 0.956 & 0.991 & 0.925 & 0.923 & 0.968 & 0.925 & 1 & 0.954 & 0.982 \\
\hline 10 & 0.935 & 0.928 & 0.961 & 0.950 & 0.941 & 0.938 & 0.93 & 0.944 & 0.954 & 1 & 0.967 \\
\hline Reference & 0.95 & 0.987 & 0.972 & 0.985 & 0.96 & 0.957 & 0.989 & 0.962 & 0.982 & 0.967 & 1 \\
\hline
\end{tabular}

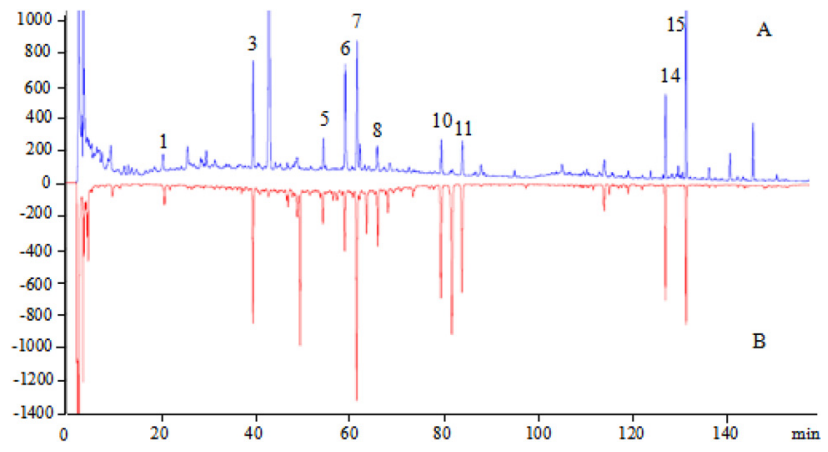

Figure 6. HPLC fingerprint chromatograms, recorded at $220 \mathrm{~nm}$, of A) a typical Xinfeng capsule sample, and B) astragalus. The peaks marked with $1,3,5,6,7,8,10,11,14,15$ are belong to astragalus. The chromatography conditions are described in Section Apparatus and chromatographic conditions

there were ten peaks simultaneously in common chromatogram belonging to Astragalus herb, indicated that Astragalus herbs played an important role in the Xinfeng capsules.

\section{CONCLUSIONS}

This method has been successfully applied for the determination of three active compounds in 10 samples. Validation results demonstrated that the developed method was sufficiently reliable and sensitive to evaluate the quality of Xinfeng Capsule. In addition, the HPLC fingerprint of Xinfeng Capsule was successfully established for the first time. Ten batches of preparations were assessed by chromatographic fingerprint analysis in combination with Similarity Analyze. These results demonstrated that the established method was a powerful and meaningful tool to comprehensively conduct the quality control of Xinfeng Capsule. These findings also provided a strong basis for the establishment of a perfect quality evaluation system of Xinfeng capsule.

\section{ACKNOWLEDGMENTS}

This work was supported by National Science and Technology Pillar Program during the Twelfth Five-year Plan
Period about study on evaluation of screening Xin'an Medicine Empirical prescription and Folk prescription (2012BA126B03), An'hui province Xin'an 115 pharmaceutical research and development innovation team. The authors are grateful to all teachers of State administration of traditional Chinese medicine TCM preparation level 3 laboratory for excellent working conditions.

\section{REFERENCES}

1. Brennan, F. M.; McInnes, I. B. J. Clin. Invest. 2008, 118, 3537-45. 2. Scott, D. L.; Wolfe, F.; Huizinga, T. W. J. Lancet. 2010, 376, 1094-108.

3. Cao, Y. X.; Liu, J.; Zhu, Y. Chin. J. Integr. Med. 2011, 17, 738-43.

4. Park, Y. J.; Kim, J. Y.; Park, J.; Choi, J. J.; Kim, W. U.; Cho, C. S. Arthritis Rheumatol. 2014, 66, 1450-60.

5. Wang, F.; Meng, M.; Chen, L.; Wang, X.; Sun, Q. JPC-J. Planar Chromat. 2014, 27, 199-203.

6. National pharmacopoeia committee. Chinese Pharmacopoeia-Part 1. 2015, 302, 357-76.

7. Liu, J.; Huang, C. B.; Wang, Y.; Xu, G. Q.; Cheng, Y. Y.; Feng, Y. X.; Liu, L.; Qi, Y. J. J. Integr. Med. 2013, 11, 428-34.

8. Xie, P. S.; Chen, S. B.; Liang, Y. Z.; Wang, X. H.; Tian, R. T.; Upton, R. J. Chromatogr. A. 2006, 1112, 171-80.

9. Kang, J.; Zhou, L.; Sun, J.; Han, J.; Guo, D. A. J. Pharm. Biomed. Anal. 2008, 47, 778-85.

10. Chen, J.; Gao, J.; Sun, G. J. Sep. Sci. 2017, 40, 1244-53.

11. Zhao, W.; Sun, F.; Cui, J.; Ren, Y.; Liu, M.; Zhao, F. Chromatographia. 2018, 81, 1293-304.

12. Sun, J.; Tian, F.; Zhang, Y.; Wu, M. H.; Mao, R. Q.; Le, Z. Y.; Xu,D. J.; Cao, H.; Ma, Z. G. Int. J. Anal. Chem. DOI: 10.1155/2019/4531092.

13. Zhang, Y.; Ding, Y.; Zhang, T.; Jiang, X. Y.; Yi, Y. X.; Zhang, L. J.; Chen, Y.; Li, T.; Kang, P.; Tian, J. J. J. Chromatogr. Sci. DOI: 10. 1093/chromsci/bmz060.

14. Tang, D. Q.; Li, Z.; Jiang, X. L.; Li, Y. J.; Du, Q.; Yang, D. Z. J. Sep. Sci. 2014, 37, 2131-7.

15. Ebrahimi-Najafabadi, H.; Kazemeini, S. S.; Pasdaran, A.; Hamedi, A. Phytochem. Analysis. 2019, 30, 405-14.

16. Lin, C. Z.; Liu, F. L.; Zhang, R. J.; Liu, M. T.; Zhu, C. C.; Zhao, J.; Li,S. P. J. Aoac Int. 2019, 102, 714-9.

17. Ofori, H.; Hettiarachchi, D.; Sostaric, T.; Busetti, F.; Boyce, M. C. JPC-J. Planar Chromat. 2019, 32, 205-10. 
18. Srivastava, M.; Maurya, P.; Mishra, S.; Kumar, N.; Shanker, K. JPCJ. Planar Chromat. 2019, 32, 211-22.

19. Custers, D.; Canfyn, M.; Courselle, P.; De Beer, J. O.; Apers, S.; Deconinck, E. Talanta. 2014, 123, 78-88.

20. Liu, Q. T.; Kong, D. D.; Luo, J. Y.; Kong, W. J.; Guo, W. Y.; Yang, M. H. J. Sep. Sci. 2016, 39, 2517-26.

21. Qing, W. W.; Shi, Y. T.; Yang, L.; Zhang, R. T.; Zhang, J. Q.; He, D. Se $p u=$ Chin. J. Chromat. 2019, 37, 1235-40.

22. Sun, Y.; Guo, T.; Sui, Y.; Li, F. J. Chromatogr. B. 2003, 792, 147-52.

23. Sun, Y. Q.; Sun, G. X.; Jin, Y. Se pu = Chin. J. Chromat. 2008, 26, $160-5$.

24. Zhou, H.; Lv, X. T.; Sun, Y. Y.; Quan, Y. K.; Guo, B. Y. J. Chromatogr. Sci. 2014, 52, 271-6.

25. Liu, Y.; Ojamae, L. J. Mol. Model. 2014, 20, 2881-8.

26. Qin, H. L.; Deng, A. J.; Du, G. H.; Wang, P.; Zhang, J. L.; Li, Z. H. J. Integr. Plant Biol. 2009, 51, 537-44.

27. Carichon, M.; Pallet, N.; Schmitt, C.; Lefebvre, T.; Gouya, L.; Talbi, N.; Deybach, J. C.; BEAUNE, P.; Vasos, P.; Puy, H. Anal. Chem. 2014, 86, 2166-74.

28. Panda, A.; Mehta, B. B.; Coppo, S.; Jiang, Y.; Ma, D.; Seiberlich, N.; Griswold, M. A.; Gulani, V. Curr. Opin. Biomed. Eng. 2017, 3, 56-66.
29. Mehta, B. B.; Coppo, S.; McGivney, D. F.; Hamilton, J. I.; Chen, Y.; Jiang, Y.; Ma, D.; Seiberlich, N.; Gulani, V.; Griswold, M. A. Magn. Reson. Med. 2019, 81, 25-46.

30. Atherton, T.; Croxton, R.; Baron, M.; Gonzalez-Rodriguez, J.; Gamiz-Gracia, L.; Garcia-Campana, A. M. A. J. Sep. Sci. 2012, 35, 2994-9.

31. Yagnik, G. B.; Korte, A. R.; Lee, Y. J. J. Mass Spectrom. 2013, 48, $100-4$.

32. Feng, S. L.; Hu, F. D.; Zhao, J. X.; Xu, J. W.; Chen, L. R. Chinese Chem. Lett. 2004, 15, 1335-8.

33. Huang, H. L.; Liu, M.; Chen, P. Anal. Lett. 2014, 47, 1835-51.

34. Sun, S. S.; Li, Y. C.; Zhu, L. J.; Ma, H. Y.; Li, L. P.; Liu Y. F. J. Sep. Sci. 2019, 42, 2875-82.

35. Duan, B. Z.; Huang, L. F.; Chen, S. L. J. Sep. Sci. 2012, 35, 513-8.

36. Li, Y. H.; Zhang, Y. M.; Zhang, Z. J.; Hu, Y. P.; Cui, X. M.; Xiong, Y. Molecules. 2019, 24, 1521.

37. Zhou, D.; Yang, Q.; Yu, Z.; Chang, Y.; Tian, T.; Shi, Z. H.; Dong, H. L.; Li, H.; Guo, J.; Wang, S. S. Iranian J. Pharm. Res. 2019, 18, 948-60.

38. Zhou, S. T.; Luan, K.; Ni, L. L.; Wang, Y.; Yuan, S. M.; Che, Y. H.; Yang, Z. Z.; Zhang, C. G.; Yang, Z. B. Molecules. 2019, 24, 2920. 